Running head: POSTURAL FEEDBACK AND CREATIVE THINKING

\title{
Do Open or Closed Postures Boost Creative performance? The Effects of Postural Feedback on Divergent and Convergent Thinking
}

\author{
Nicolas Michinov and Estelle Michinov
}

University of Rennes 2

(C) 2020, American Psychological Association. This paper is not the copy of record and may not exactly replicate the final, authoritative version of the article. Please do not copy or cite without authors' permission. The final article will be available, upon publication, via its DOI: 10.1037/aca0000306 in Psychology of Aesthetics, Creativity, and the Arts

\section{Author Note}

Special thanks to research assistants for their help in collecting or coding data for this research: Nolwenn Barré, Lisa Bezier, Malvina Bloux, Elaura Briand, Enora David, Sasha Donnier, Amélie Ferré, Rozenn Finas, Steven Floch'Lay, Aurélia Gauchet, Camille Gillet, Mégane Guellec, Rozenn Guernier, Wali Hammoudi, Oumaïma Hathroubi, Emilie Havart, Mathilde Hervé, Jenna Kerdranvat, Marie-Laure Kandor, Christopher Octobre, Lucas Sabatier, Louisa Tellal, Victoire Veillé, Sophie Verger, and Mathis Vienne. A very special thanks to Steven Floch'Lay for the drawings of androgynous persons in expansive and contractive postures.

The authors have no competing interests to declare, and this research did not receive any specific grant from funding agencies in the public, commercial, or not-for-profit sectors. 
Data and supplemental materials are available on our OSF project page: $\underline{\text { osf.io/c9mzh }}$

Correspondence concerning this article should be sent to the following address:

Nicolas Michinov and Estelle Michinov, Laboratoire de Psychologie: Cognition,

Comportement, Communication (LP3C), Université Rennes 2, place du Recteur Henri Le

Moal, 35043 Rennes Cedex, France. E-mail: nicolas.michinov@univ-rennes2.fr and

estelle.michinov@univ-rennes2.fr 


\begin{abstract}
Can an individual's body posture (expansive or contractive) affect their creative thinking (divergent or convergent)? Based on embodied cognition and the debate about the impact of nonverbal physical postures expressing power on psychological and behavioral outcomes, five experiments were conducted. We tested the prediction that expansive postures would have a positive effect on creativity tasks that have no right or wrong answer or optimal solution (divergent thinking), whereas contractive postures would have a positive effect on tasks with a right answer or an optimal solution (convergent thinking). As predicted, results revealed a positive effect of expansive postures on performance of creativity tasks requiring divergent thinking, such as producing original ideas (Study 1) or objects, either by combining shapes to create an original toy (Study 2) or by combining fragments to produce an original drawing (Study 3). Conversely, a positive effect of contractive postures was found on performance of insight tasks requiring convergent thinking, in which participants had to associate elements to discover a unifying and correct solution (Study 4) or overcome initial task constraints to find an optimal solution to a problem (Study 5). These findings open up new avenues for research in embodied creativity.
\end{abstract}

Keywords: postural feedback; power; creative thinking; embodied creativity; nonverbal behavior 


\section{Do Open or Closed Postures Boost Creative performance? The Effects of Postural Feedback on Divergent and Convergent Thinking}

Since the pioneering work of James (1894), considerable research on embodied cognition has examined the influence of the body's physical states on psychological, behavioral, and physiological outcomes (e.g., Barsalou, 2008; Niedenthal, Barsalou, Winkielman, KrauthGruber, \& Ric, 2005). In the last ten years, a growing body of research has focused specifically on the impact of nonverbal physical postures expressing power and dominance on various psychological, emotional and behavioral outcomes (e.g., Carney, Cuddy, \& Yap, 2010; Cesario \& McDonald, 2013; Yap, Wazlawek, Lucas, Cuddy, \& Carney, 2013). Postural expansion and contraction are nonverbal cues reflecting high and low dominance and power both in humans and in many animal species (Metzler, 2018). Expansive body postures signal high dominance or power, whereas constrictive postures convey low power (Hall, Coats, \& LeBeau, 2005). Different manipulations of power by priming procedures (or recalling a situation of power) create a sense of power that then produces a range of cognitive, behavioral, and physiological consequences (Galinsky, Rucker, \& Magee, 2015). Specifically, research has suggested that power contributes to increase creativity (Mehta \& Dahl, 2019), leading individuals to exhibit cognitive flexibility changing their ways of thinking creatively (Overbeck \& Park, 2006; Yin \& Smith, 2020). It also appeared that manipulating bodily postures expressing power may have an impact on abstract thoughts and feelings, but empirical evidence remains limited on divergent creative thinking (Andolfi, Di Nuzzo, \& Antonietti, 2017; Hao, Xue, Yuan, Wang, \& Runco, 2017). Thus, the aim of the present research was to examine whether open-expansive postures (presumed to create a state of high power) could have a positive effect on divergent (or open-ended) creative thinking, and conversely, whether closed-contractive postures (presumed to create a state of low power) could have a positive effect on convergent (or close-ended) creative thinking. 


\section{Effects of Body Posture on Outcomes}

Although previous research used upright and slumped sitting postures as bodily feedback manipulations (Riskind, 1984; Riskind \& Gotay, 1982), Cuddy, Schultz, and Fosse (2018) recently introduced the concept of "postural feedback" to characterize behaviors consisting of holding certain open-expansive postures (or closed-contractive postures) during a short time period and evaluated their impact on various outcomes in different contexts. Using the initial two-step paradigm, first inducing postures and then measuring outcomes (Carney et al., 2010), researchers found that holding expansive postures (versus contractive postures) had positive effects on a wide range of outcomes, such as feeling of power (Bombari, Schmid Mast, \& Pulfrey, 2017; Cuddy et al., 2018; Gronau, Van Erp, Heck, Cesario, Jonas, \& Wagenmakers, 2017; Park, Streamer, Huang, \& Galinsky, 2013; Ranehill, Dreber, Johannesson, Leiberg, Sul, \& Weber, 2015), implicit activation of power (Huang, Galinsky, Gruenfield, \& Guillory, 2011), pain tolerance (Bohns \& Wiltermuth, 2012), selfesteem (Körner, Petersen, \& Schütz, 2019), job interview performance (Cuddy, Wilmuth, Yap, \& Carney, 2015), anti-social behaviors (Meier, Schöbel, \& Feufel, 2019; Yap et al., 2013), creative thinking (Andolfi et al., 2017; Hao et al., 2017), etc.

Recently, a number of criticisms have emerged regarding the robustness of this "power posing" effect, many studies failing to replicate or to demonstrate the positive effects of holding expansive postures on various outcomes (see Jonas, Cesario, Alger, Bailey, Bombari, Carney et al., 2017), such as hormonal changes (Metzler \& Grèzes, 2019; Ranehill et al., 2015; Ronay, van Huijstee, \& Morssinkhof, 2017; Smith \& Apicella, 2017), risk-taking behavior (Cesario \& Johnson, 2017; Bailey, LaFrance, \& Dovidio, 2017), and gambling decisions (Bombari et al., 2017). A lively scientific debate has subsequently developed about the effects (or lack of effect) of body posture, based on various methodological and statistical 
arguments (Carney, Cuddy, \& Yap, 2015; Cesario, Jonas, \& Carney, 2017; Credé \& Phillips, 2017; Cuddy et al., 2018; Simmons \& Simonsohn, 2017). On the one hand, Credé and Phillips (2017) argued that the findings about "power posing" depend on the method used to select participants and detect outliers, and the strategy used to choose dependent measures and control variables. On the other hand, strong statistical arguments have been advanced to explain the effect (or the absence of effect) of "power posing" on behavior. Based on a pcurve analysis of the 33 studies reviewed by Carney and collaborators (2015), Simmons and Simonsohn (2017) found a lack of empirical support for the behavioral and physiological effects of postural feedback. In response, using the same p-curve analysis strategy on 55 studies, Cuddy et al. (2018) provided arguments demonstrating that expansive postures have a robust impact on the feeling of power and EASE variables (Emotion, Affect, and SelfEvaluation), in contrast to contractive postures. By contrast, the effects were less convincing for non-EASE variables grouping a large variety of behaviors such as risk-taking, thought abstraction, pain tolerance, hormonal changes, eating behavior, and creativity.

With these considerations in mind, the present research aimed to add to this debate, seeking to understand why different effects (or no effect) may be observed on non-EASE variables, and more specifically on creativity. In this research field, two creative thinking processes - divergent and convergent - have been identified in the literature (Guilford, 1950). Divergent thinking is the ability (or potential) to generate multiple ideas or solutions to an open-ended problem that does not have a right or wrong answer. In other words, divergent thinking consists of finding many possible solutions by approaching the problem from different directions, for example when responding to open-ended questions that do not have right or wrong answers (e.g., Alternative Uses Task, Guilford, 1967; Parallel lines task, Torrance, 1974), or by combining different elements to produce original drawings (e.g., Creative Invention Task, Finke, 1990; Test of Creative Thinking-Drawing Production, Urban 
\& Jellen, 1996). By contrast, convergent thinking is the ability (or potential) to apply conventional and logical information search, recognition, and decision-making strategies to find creative solutions to close-ended problems having only one correct answer, such as insight problems. These problems generally refer to the 'associative view of creativity', suggesting that creativity results from active unconscious processes (e.g., Beaty, Nusbaum, \& Silvia, 2014; Schooler, Ohlsson, \& Brooks, 1993). For insight problems, creative thinking consists of finding the right and original answer to a problem because the first and most obvious solution is often wrong. One of the most widely used (verbal) insight problems consists of giving participants three apparently unrelated words and asking them to think of a fourth word that is related to each of them (RAT, Remote Associates Test, Mednick, 1962). Other problems consist of giving the participants some contextual information about a problem, and then asking them a question that requires shifting their perception of the problem and viewing it in a new way in order to find the solution (e.g., Dow \& Mayer, 2004; Duncker, 1945; Maier, 1930).

\section{Embodied Creativity Research}

In the field of embodied creativity, the relationships between different nonverbal body cues and creativity have received increasing interest in recent years (Frith, Miller, \& Loprinzi, 2019; Malinin, 2019; Stanciu, 2015). For instance, body movements have been shown to be associated with creative thinking (e.g., Oppezzo \& Schwartz, 2014; Zhou, Zhang, Hommel, \& Zhang, 2017) and are recognized as having an impact on performance of both divergent and convergent thinking tasks. For example, Oppezzo and Schwartz (2014, Study 1) found that participants performed better on a divergent thinking task (Alternative Uses Task) when they walked than when they remained seated. Conversely, walking reduced the number of correct answers in a convergent thinking task (Remote Associates Test). The relationships between gestures and creative thinking have also been examined in the literature (e.g., Friedman \& 
Förster, 2000; 2002). For example, arm flexion and extension can activate different information-processing motivations (approach or avoidance) impacting creativity. In a series of studies (Friedman \& Förster, 2000), it has been demonstrated that arm flexion (versus extension) facilitated insight on tasks involving convergent thinking processes, such as the embedded figure task, which consists of identifying figures hidden in complex visual patterns (Witkin, Oltman, Raskin, \& Karp, 1971). In another study where hand gestures were manipulated (Slepian \& Ambady, 2012), participants who had to trace the most fluid drawing (a round, continuous snake-like drawing) showed better results in creativity tasks requiring divergent thinking, and solved fewer insight problems (RAT) requiring convergent thinking. Finally, studies have examined the relationships between posture, feelings of power, and creative thinking. Some found that expansive postures increased the feeling of power (Bombari et al., 2017; Cuddy et al., 2018; Gronau et al., 2017), and others showed that provoking a feeling of power by different procedures (e.g., priming or recalling a situation of power) had an influence on creative divergent thinking (Friedman, Fishbach, Förster, \& Werth, 2003; Galinsky, Magee, Gruenfeld, Whitson, \& Liljenquist, 2008; Gervais, Guinote, Allen, \& Slabu, 2013; Sligte, De Dreu, \& Nijstad, 2011).

Recently, researchers have started to examine whether the influence on divergent thinking of briefly holding an expansive posture could be similar to recalling or being primed by a power situation (Andolfi et al., 2017; Hao et al., 2017). They found that participants holding expansive-open postures while performing the Alternative Uses task verbally generated more unusual ideas than those in contractive-closed postures (Andolfi et al., 2017). Similar findings were observed on two sub-tasks of the Torrance Tests of Creative Thinking (Torrance, 1974), both measuring divergent thinking (Product Improvement Task and Just Suppose Task). In another study, Hao et al. (2017) found similar outcomes on creativity tasks based on divergent thinking, such as the Realistic Presented Problem, but only when positive 
emotions were induced among participants before adopting expansive postures. Taken together, these studies indicate some benefits of expansive-open postures in creativity tasks requiring divergent thinking. However, the initial two-step paradigm, first inducing postures and then measuring creative performance, was not used in these studies; instead individuals had to perform a creative task while adopting an expansive or contractive posture. Moreover, only creativity tasks requiring divergent thinking were used in previous studies (see Malinin, 2019), while creativity tasks requiring convergent thinking have not been investigated to date.

\section{Present Research and Hypotheses}

The aim of the present research was to examine through a series of studies whether expansive postures expressing power might improve creative performance in various tasks requiring divergent thinking due to a holistic (or global) mode of processing information. Conversely, we also examined whether contractive postures expressing low power might improve creativity in tasks requiring convergent thinking due to a detailed, analytical (or local) mode of processing information. Although the link between power, creativity and information processing has been examined in the literature (Friedman et al., 2003; Gervais et al., 2013; Keltner, Gruenfeld, \& Anderson, 2003), the feeling of power was generally not operationalized by postures, and the present research aimed to fill this gap.

Different experimental procedures were used to induce a feeling of power; at least two of these, priming and recall of a situation of power, are known to have an influence on creative performance due to different types of information processing. A study that manipulated the feeling of power through a priming procedure found that participants produced more creative responses (Galinsky et al., 2008, Study 1). In another study that manipulated the feeling of power by asking participants to recall a situation in which they had power over another person, participants who recalled a situation of power were more creative 
than those who did not (Gervais et al., 2013; Keltner et al., 2003). It has been suggested that the distinction between holistic (or global) and analytical (or local) processing can explain the differences in creative performance: powerful people are better able to adopt a more global attentional focus, while powerless individuals adopt a local attentional focus (Guinote, 2007; Smith \& Trope, 2006). Consequently, the feeling of power may enhance creative performance in tasks requiring divergent thinking because it facilitates a global attentional focus. Other studies found that when individuals had to complete visual tasks that forced them to focus their attention on either a broad or a narrow visual area, broad focusing of perceptual attention led to more original and unusual ideas (Friedman et al., 2003).

Based on these previous studies, a strong sense of power induced by expansive postures should lead individuals to adopt a holistic mode of information processing, leading to greater creative performance on tasks that do not have a right or wrong answer, in other words, requiring divergent thinking. Conversely, as individuals with a weak sense of power have better insight performance (Sligte et al., 2011), contractive postures should lead to more creative performance on tasks requiring convergent thinking, such as insight problems (e.g., Dow \& Mayer 2004; Metcalfe \& Wiebe, 1987; Schooler \& Melcher, 1995). In other words, the weak sense of power induced by contractive postures should lead individuals to have a narrow attentional focus (Smith \& Trope, 2006) and to examine different and unexpected angles to find the correct solution to tasks requiring convergent thinking.

To test these predictions, five experiments were conducted using the same two-step procedure; first, the posture of participants was manipulated to induce different levels of power, and secondly, they were asked to carry out tasks requiring different creative thinking processes in order to measure creative performance. In contrast to previous research in which a verbal creativity task was performed while participants adopted a given posture (Andolfi et 
al., 2017; Hao et al., 2017), the two-step paradigm was chosen in the present study because it is consistent with the postural feedback literature examining the effects of physical postures expressing power on various psychological and behavioral outcomes (e.g., Carney et al., 2010; Cesario \& McDonald, 2013; Yap et al., 2013). In all the studies, participants were instructed to hold a series of four expansive or contractive postures illustrated in a leaflet for 3 minutes, before performing a creativity task lasting 15 minutes. A different task was used in each experiment to test the effect of posture on creative performance. The first three experiments used creativity tasks requiring divergent thinking, such as producing ideas (Study 1; AUT, Alternative Uses Task; Guilford, 1967) or original objects, either by combining shapes to create a toy (Study 2; CIT, Creative Invention Task, Finke, 1990; Finke, Ward, \& Smith, 1992) or by combining fragments to produce a drawing (Study 3; TCT-DP, Test of Creative Thinking Drawing Production, Urban \& Jellen, 1996). The following two experiments used creativity tasks requiring convergent thinking, such as insight tasks, in which participants had to associate elements to discover a unifying and correct solution (Study 4; RAT, Remote Associates Test; Mednick, 1962) or overcome initial task constraints to find an optimal solution to a problem (Study 5; insight problems; Dow \& Mayer 2004). All procedures in these studies were in accordance with the ethical standards of institutional and/or national research committees for studies involving human participants and with the 1964 Helsinki Declaration and its later amendments or comparable ethical standards. Participants completed informed-consent forms and were debriefed after each experiment, when they were told the objectives and procedures. In the absence of an institutional research ethics committee at the university, the vice president of research, the scientific integrity advisor, and the personal data protection officer examined the research project and gave a favorable opinion. They judged the project to comply with usual practices and ethical rules in 
the human and social sciences and issued a certificate of ethical approval for the present research.

\section{Study 1: Effects of Postures on an Idea-Generation Task}

\section{Method}

\section{Participants}

We determined the required sample size using G*Power (Faul, Erdfelder, Lang, \& Buchner, 2007; Perugini, Gallucci, \& Costantini, 2018). As previous research has demonstrated the effect of posture on creative thinking (Andolfi et al., 2017), we assumed a 'medium' effect size $(d=0.60)$. Power analysis test indicated an optimal sample of 72 (36 participants per cell), based on $\alpha=.05$ and power $=0.80$ for a one-tailed test (and 90 participants for a two-tailed test). As theoretical hypotheses based on the effect of postural feedback are clearly oriented in one direction, we used one-tailed tests in the present research.

Seventy-six participants (51 females and 25 males), aged 17 to 24 years $(M=19.53$, $S D=1.59$ ), were randomly assigned to either the expansive or the contractive posture condition (38 participants in each condition). A Chi-square test revealed no significant difference in the distribution of males and females between the two conditions, $\chi^{2}(1, N=76)$ $=.060, p=.807, \phi=.028$. All the participants were undergraduate or graduate students in a medium-sized French university in various academic disciplines: psychology $(N=23)$, arts and languages $(N=13)$, humanities $(N=14)$, art and architecture $(N=9)$, law, administration, accounting, communication $(N=8)$, sports science $(N=4)$, STEM $(N=4)$, and learning sciences $(N=1)$. They were recruited by research assistants on the campus and were told that they would participate in an ergonomic study about the effects of sitting posture in the workplace. 


\section{Procedure}

The procedure followed the initial two-step paradigm, in which participants first adopted a series of postures and then completed the outcome measures (Carney et al., 2010). After reading and signing a consent form, participants were informed by the experimenter (one of the two research assistants, 1 male and 1 female) that the objective of the study was to collect data about the impact of the ergonomic quality of an office chair with arms on the quality of work. They were told the following cover story, inspired by the one used by Park and collaborators (2013, Study $2 \mathrm{~b}$ and Study 3 ): The purpose of this ergonomic study is to examine the effect of sitting posture on the quality of work. The objective is to evaluate different ways of sitting in an office chair. As the quality of office chairs can make individuals adopt certain postures rather than others, they can also have an influence on work performance. Therefore, we wish to test a few sitting postures that you will hold for a short time to evaluate their impact on performance of a cognitive task. The main goal is to improve the working conditions of office workers.

The experimenter then instructed them to sit in the office chair adopting four postures presented in a leaflet, in a set order (see Appendix). The drawings of androgynous persons in expansive and contractive postures were created by one of the research assistants. Six black and white drawings of people wearing similar clothes and without facial expression in different postures were inspired by the Chapter 8 of Cuddy's (2015) book (expansive postures no. 2, 3, 5; contractive postures no. $7,9,10$ ). Male and female attributes of the figures were removed in order to control for gender, a potential moderating variable that has recently been identified in the literature (Bailey et al., 2017; Bombari et al., 2017; Latu, Mast, Bombari, Lammers, \& Hoyt, 2018). Two other postures were included, so that there were four sitting postures in each condition ${ }^{1}$. Two leaflets were created, one with expansive and one with 
contractive postures (see N. Michinov \& E. Michinov, 2020 for the Online Supplementary Materials 1 and 2). They were randomly distributed among participants so that the experimenter was blind to the experimental condition. The experimenter left the room while the participants posed as in the illustration. They were instructed that they should change posture every 45 seconds, on hearing a pre-recorded signal. The maximum recommended time is one minute per posture (Carney et al., 2015, p. 6), but because there were four postures in each condition, the duration per posture was reduced by 15 seconds (i.e. a total of 3 minutes for the four postures). This reduction was important to avoid discomfort, especially in the expansive 'feet-on-the-desk' posture.

After this phase, a synthetic vocal message instructed participants to move to an adjacent table, and to sit on a static chair without arms to perform the cognitive task, following the instructions on a sheet of paper placed upside down on the table. They were told that they had 15 minutes to think of as many different ways as possible to use a cardboard box and write them down on a piece of paper with numbered lines, one idea per line.

During the task, the experimenter watched from behind a window with a venetian blind to check that participants followed the instructions, assumed the correct postures, and respected the timing. Based on these observations, it appeared that all participants followed the instructions adopting the four postures mentioned in the leaflets. This procedure was preferred to video recording, which could have distracted participants from the task and modified their spontaneous behavior. At the end of the 15 minutes, the experimenter returned and gave the participants a one-page questionnaire including perception of the task, sociodemographic information, and a manipulation check. After completing the questionnaire, participants were debriefed and thanked. If they wished, they could also give their email address in order to receive information about the results of the study. 


\section{Measures}

\section{Manipulation check}

A figure containing the eight posture drawings was included in the post-experimental questionnaire. Participants had to circle the postures they had adopted before the cognitive task. They were given a score of 1 when all the postures were correctly recognized, and 0 in the other cases.

\section{Creative performance}

Creative performance was measured by fluency/fluidity, i.e. the number of ideas produced by each participant. Fluency is one of the indicators of divergent thinking, easy to measure; it is generally related to flexibility (i.e. number of categories of ideas), and originality (i.e. score of novelty of ideas produced). The flexibility score was computed from a 'master list' of ideas extracted from previous experiments (e.g., Michinov, Jamet, Métayer, \& Le Hénaff, 2015), in which inter-coder reliability was good for the ten categories of ideas identified (storage of objects; animal habitats and food; games; arts and decoration; musical instruments; do-it-yourself and repair; use of cardboard; utensils, objects or accessories;

gardening; clothes and disguises). The total number of categories explored by each participant was used as a measure of flexibility. For each participant, we extracted from the same 'master list' an originality score from the mean of originality of ideas produced by two coders, ranging from 1 (very common idea) to 5 (very original idea). For example, a participant who produced five ideas rated $[1.5 ; 2.5 ; 3.0 ; 4.0 ; 3.5]$ were summed $[\mathrm{Sum}=15.5]$ and divided by five to determine the mean originality of his/her ideas [Mean originality $=2.90]$. The three measures of creativity were inter-correlated (fluency and flexibility, $r=.562, \mathrm{p}<.001$; fluency and originality, $r=.368, \mathrm{p}<.001$; flexibility and originality, $r=.613, \mathrm{p}<.001$ ). 


\section{Results and discussion}

Descriptive analyses to check the efficacy of the experimental manipulation indicated that 68 participants out of $76(89.5 \%)$ correctly recognized the four postures they had adopted, $92.1 \%$ in the contractive posture condition, and $86.8 \%$ in the expansive posture condition. A chi-square test revealed no significant difference between the two conditions, $\chi^{2}(1, N=76)=$ $.559, p=.455, \phi=-.086$.

As predicted, a one-tailed test revealed that more ideas were produced by participants in the expansive posture condition $(M=15.97$ and $S D=7.35)$ than by those in the contractive posture condition $(M=12.10$ and $S D=4.51$; mean difference $=-3.868,95 \%$ confidence interval, or CI $=[-\infty,-1.080]), t(74)=-2.765, p=.004$, Cohen's $d=0.634$. A similar test revealed no significant difference between conditions for flexibility (mean difference $=$ $0.500,95 \%$ confidence interval, or $\mathrm{CI}=[-\infty, 0.162]), t(74)=-1.504, p=.068$, Cohen's $d=$ 0.34 ), and originality (mean difference $=-0.052,95 \%$ confidence interval, or $\mathrm{CI}=[-\infty$, $0.092]), t(74)=-0.718, p=.24$, Cohen's $d=0.16)$. No significant effect was found on the post-experimental measures ${ }^{2}$.

The results demonstrated that participants who adopted expansive postures for a few minutes before performing an open-ended creativity task generated more ideas than those in contractive postures. In other words, adopting expansive-open postures for a short-time predisposed participants to be creative when the task required divergent thinking processes, and they showed greater fluency than those in contractive postures. These results extend findings reported in the literature using a different procedure (Andolfi et al., 2017; Hao et al., 2017); in those studies, participants had to generate ideas verbally while they were sitting in either an expansive or a contractive posture. By contrast, in the present study, participants had to write their ideas down after sitting in four successive postures, either expansive or 
contractive, for 3 minutes, as in the initial two-step paradigm (Carney et al., 2010; Cuddy et al., 2015; Park et al., 2013).

Although the number of ideas produced by participants (fluency) was correlated with the number of categories (flexibility) and originality of ideas, the postures did not have a significant impact on the two latter measures. As fluency is the most relevant indicator of divergent thinking in the creativity literature, it is not surprising that the positive impact of expansive postures was only observed on the quantity, and not on the quality of ideas. Our findings are consistent with previous research in this field demonstrating that participants holding expansive-open postures have better creative performance on the 'Alternative uses task' (Andolfi et al., 2017). Similar findings have also been found on creative performance on two verbal sub-tasks of the Torrance Tests of Creative Thinking (Andolfi et al., 2017), the 'Product Improvement task', which involves listing possible improvements for a product (e.g., a stuffed toy elephant), and the 'Just Suppose task', in which participants have to describe all the things that might happen if an improbable situation occurred (e.g., clouds with strings that hang down to the earth). In the present study, asking the participants to sit for a short time in expansive postures led them to process information holistically and enabled them to be more creative on a subsequent task requiring divergent thinking.

As this study, like previous ones, focused on verbal divergent thinking tasks, we wished to explore whether the effect of postural feedback could be extended to other types of creativity tasks, such as figural tasks requiring divergent thinking. In these tasks, there was no correct or optimal solution, and participants had to combine different shapes to invent an original object.

Study 2: Effects of Postures on a Creative Invention Task 


\section{Method}

Participants and overview of the procedure

Seventy participants (52 females and 18 males), aged 18 to 26 years $(M=20.67, S D=$ 1.95), were randomly assigned to either an expansive or a contractive posture condition. A Chi-square test revealed no significant difference in the distribution of males and females between the two conditions, $\chi^{2}(1, N=70)=1.197, p=.274, \phi=.131$. All the participants were students in various disciplines, mostly in psychology $(N=45)$, the others in arts and languages $(N=10)$, law, administration, accounting, communication $(N=4)$, sports science $(N=4), \operatorname{STEM}(N=4)$, and humanities $(N=3)$.

A similar procedure to the previous study was used, except that participants had to perform a figural creativity task based on the Creative Invention Task (CIT, Finke, 1990; Finke et al., 1992). In this task, participants were asked to imagine an original toy for 5- to 11 year-old children and to draw it on an A5 format (5.83 x 8.27 inches) erasable whiteboard, by combining five amongst 20 possible different shapes (see Mehta \& Zhou, 2009, study 4). They had 15 minutes to draw their invention using each shape once. At the end of the task, the experimenter (one of the two female research assistants) came in and gave participants the same questionnaire as in the first study. While they completed it, their drawing was copied to be rated by different coders. After completing the questionnaire, participants were debriefed and thanked.

\section{Measures of creative performance}

The drawings were rated on originality by four independent coders using the sevenpile "sort-resort" technique rather than Amabile's (1982) consensual assessment technique, which is often used in the creativity literature. The "sort-resort" technique was adapted from 
Shaw (1963) by Hackman et al. (1967). Since then, it has been used in several studies on individual and group creativity to assess the originality of different productions such as products, ideas, slogans, tasks, etc. (e.g., Beersma \& De Dreu, 2005; Craig \& Kelly, 1999; Jones \& Kelly, 2009; Kelly \& Karau, 1993). Each coder received a folder containing copies of all the drawings presented in random order. The coders did not know the purpose of the experiment or the experimental condition to which participants had been allocated. They sorted the drawings by originality (defined as the unusual and unique aspect of the object) into three piles (high, medium, and low originality). Each pile was then re-sorted. The "high" pile was sorted into two piles (high-high and high-low), the "medium" pile was sorted into three piles (medium-high, medium, and medium-low), and the "low" pile was sorted into two piles (low-high and low-low). This resulted in seven piles corresponding to a 7-point originality scale ranging from 1 (low) to 7 (high). As the inter-coder reliability was satisfactory $(\mathrm{ICC} 2=.66)$, a single composite score was used to assess creativity based on the mean scores of the four coders (see Amabile, 1982). The same 'sort-resort' technique was applied to evaluate the complexity of the object $(\mathrm{ICC} 2=.58)$.

\section{Results and discussion}

Descriptive analyses to check the efficacy of the experimental manipulation indicated that 65 participants out of $70(92.9 \%)$ correctly recognized the four postures they had adopted, $97.1 \%$ in the contractive posture condition and $88.6 \%$ in the expansive posture condition. A Chi-square test revealed no significant difference between the two conditions, $\chi^{2}(1, N=70)=$ $1.938, p=.164, \phi=.166$

As predicted, a one-tailed test revealed that the originality of the drawings was higher in the expansive posture condition $(M=4.679$ and $S D=1.50)$ than in the contractive posture condition $(M=3.600$ and $S D=1.464$; mean difference $=-1.079,95 \%$ confidence interval, or 
$\mathrm{CI}=[-\infty,-0.488]), t(68)=-3.045, p=.002$, Cohen's $d=0.728$. A similar analysis on the complexity of the objects did not reveal any difference between the expansive posture condition $(M=4.164$ and $S D=1.456)$ and the contractive posture condition $(M=3.936$ and $S D=1.571 ;$ mean difference $=-0.229,95 \%$ confidence interval, or $\mathrm{CI}=[-\infty, 0.375]), t(68)=$ $-0.631, p=.265$, Cohen's $d=0.151$.

The results confirmed that participants who adopted expansive postures for a few minutes before performing a creativity task were more creative than those who adopted contractive postures and produced more original drawings. However, findings also revealed that participants in expansive postures did not produce drawings of greater complexity than those in contractive postures. Like previous findings demonstrating the positive effect of expansive postures on creativity (Andolfi et al., 2017; Hao et al., 2017), the present results suggest that expansive postures may have a positive impact on a creative task that requires divergent thinking and which is more complex than the idea-generation tasks often used to measure creativity. In other words, information was processed holistically when a feeling of power was momentarily induced by expansive postures, leading to more creative performance in a divergent thinking task. As previously, these results suggest that participants were more creative after holding expansive postures, inducing a feeling of power, than contractive postures, inducing no feeling of power.

As this task is similar to the figural sub-task of the TTCT (Torrance, 1974), which involves combining shapes to create a new object, it is reasonable to conclude that expansiveopen postures lead to more creative performance than contractive-closed postures in tasks requiring divergent thinking. As there is very little empirical evidence regarding the causal relationship between expansive postures and divergent creative thinking, we conducted a third, smaller-scale study to further validate our hypothesis, using another figural creativity 
task, the Test of Creative Thinking-Drawing Production (TCT-DP; Jellen \& Urban, 1989; Urban \& Jellen, 1996), consisting of combining fragments to produce an original drawing.

\section{Study 3: Effects of Postures on the Test of Creative Thinking-Drawing Production}

\section{Method}

Participants and overview of the procedure

Forty-four women, aged 17 to 25 years $(M=19.68, S D=1.76)$, were randomly assigned to either an expansive or a contractive posture condition. All were students in various disciplines, the majority in arts and languages $(N=12)$ and $\operatorname{STEM}(N=10)$, the others in psychology $(N=6)$, law, administration, accounting, communication $(N=5)$, art and architecture $(N=4)$, health, social work and nursing $(N=4)$, humanities $(N=2)$, and sports science $(N=1)$.

The study was conducted by two research assistants ( 1 male and 1 female) using a similar procedure to the above studies, except that participants had to perform the TCT-DP. The TCT-DP provides a scoring system using a set of criteria and has been recognized as a valid measure of creative thinking. The material consists of a square frame ( $4.72 \times 4.72$ in) drawn on a sheet of A4 paper, with six geometrical fragments, one outside the frame (a small open-sided square), and the other five inside the frame (semicircle, dot, large right angle, Sform, and dashed line). Participants are instructed to make an original drawing using these fragments. The test has two parallel forms, A and B, both containing the same elements, but Form $\mathrm{B}$ is a $180^{\circ}$ inversion of Form A. Thirty minutes are allowed to complete the two forms; as we only used Form $\mathrm{A}$ in the present study, participants were given 15 minutes to make a drawing in any way they wished using the geometrical fragments. Finally, the participants were asked to complete a brief questionnaire, before being debriefed and thanked. 


\section{Measures of creative performance}

Each drawing was evaluated by three coders blinded to the hypotheses and trained to use the coding system. The TCT-DP consists of 14 criteria that form the total score (Urban \& Jellen, 1996), applied to the completed drawing: nine conventional criteria (continuations, completions, new elements, connections made with a line, connections made to produce a theme, boundary breaking that is fragment dependent, boundary breaking that is fragment independent, perspective, humor and affectivity), and four unconventional criteria (manipulation of the material, surreal or abstract drawings, atypical combinations of figures and symbols, non-stereotypical use of a certain element). The fourteenth criterion (Speed) was not used in the present study because the time spent on the drawing was the same for all participants. Each criterion is scored using an ordinal measurement scale; a score of 0 to 6 is given for the first nine criteria, and 0 to 3 for the four unconventionality sub-criteria. An overall creativity score was produced by summing the ratings of each coder on all of the criteria. As the inter-coder reliability was highly satisfactory, $\operatorname{ICC}(2)=.87$, a single composite creativity score was produced. The total possible score range of the TCT-DP, excluding Speed, was 0-66 points. High scores indicate a high level of creativity. The inter-coder reliability was also highly satisfactory for both conventionality and unconventionality scores, $\operatorname{ICC}(2)=.87$ and $\operatorname{ICC}(2)=.67$, respectively. Despite the scoring instructions, one criterion may be confounded with another, and consequently, to be sure that overall creativity was measured correctly, the task was scored by two different coders using the 'sort-resort' technique, $\operatorname{ICC}(2)=.90$. The degree of detail in the drawings and the complexity of integration were also coded using the same technique. The inter-coder reliability was satisfactory for the first measure, $\operatorname{ICC}(2)=.80$, and poor for the second, $\operatorname{ICC}(2)=.41$.

\section{Results and discussion}


Descriptive analyses to check the efficacy of the experimental manipulation indicated that 36 participants out of $44(83.7 \%)$ correctly recognized the four postures they had adopted, $76.2 \%$ in the contractive posture condition and $90.9 \%$ in the expansive posture condition. A Chi-square test revealed no significant difference between the two conditions, $\chi^{2}(1, N=43)=$ $1.708, p=.191, \phi=.199$.

As predicted, a one-tailed test revealed that the overall creativity of drawings was higher in the expansive posture condition $(M=28.303$ and $S D=8.46)$ than in the contractive posture condition $(M=23.924$ and $S D=7.078$; mean difference $=-4.379,95 \%$ confidence interval, or $\mathrm{CI}=[-\infty,-0.424]), t(42)=-1.862, p=.035$, Cohen's $d=0.561$. The difference was only observed on the 10 conventionality criteria $(M=25.788$ and $M=21.591$, respectively; mean difference $=-4.197,95 \%$ confidence interval, or $\mathrm{CI}=[-\infty,-0.525]), t(42)$ $=-1.922, p=.031$, Cohen's $d=0.579)$, and not on the 4 unconventionality criteria $(M=2.515$ and $M=2.333$, respectively; mean difference $=-0.182,95 \%$ confidence interval, or $\mathrm{CI}=[-\infty$, $0.721]), t(42)=-0.339, p=.368$, Cohen's $d=0.102)$.

The same effect was observed on the originality measure based on the 'sort-resort' technique, and the two measures were correlated, $r=0.72, p=.001$. As for the other measures, it was found that originality was higher in the expansive posture condition $(M=$ 5.159 and $S D=1.64)$ than in the contractive posture condition $(M=3.727$ and $S D=2.080$; mean difference $=-1.432,95 \%$ confidence interval, or $\mathrm{CI}=[-\infty,-0.481]), t(42)=-2.534, p=$ .008 , Cohen's $d=0.764$. Despite poor agreement between the coders, the results revealed a significant difference on the complexity of integration, which was higher in the expansive posture condition $(M=5.205$ and $S D=1.054)$ than in the contractive posture condition $(M=$ 3.750 and $S D=1.470 ;$ mean difference $=-1.455,95 \%$ confidence interval, or $\mathrm{CI}=[-\infty,-$ 
$0.806]), t(42)=-3.772, p<.001$, Cohen's $d=1.137$. No difference was found on the degree of detail in the drawings.

Consistent with our predictions, participants who had been placed in expansive postures for a few minutes before performing the creativity task were more creative than those who had been placed in contractive postures. Once again, this study confirmed our predictions demonstrating the positive impact of expansive postures on creative performance in a complex task requiring divergent thinking. The same results were found when creativity was measured by an evaluation based on ratings on a series of criteria, but also when it was measured with the 'sort-resort' technique, on both originality and degree of integration in the drawings. However, the results for the degree of integration in the drawings should be considered with caution because the reliability between coders was poor, in contrast to coding on other measures. It can be pointed that the differences between expansive and contractive postures were observed on superficial criteria such as the degree of conventionality in each drawing, and not on richer criteria, such as unconventionality or degree of details in the drawings. These findings are consistent with those of Study 1, where the differences between the conditions were only observed on fluency, and not on flexibility or originality of generated ideas. They are also consistent with the findings of Study 2, where differences were only observed on originality of drawings, and not on complexity. This small-scale study required a sophisticated and laborious coding phase with five coders blind to the hypotheses, examining in detail each production on a set of specific criteria. The findings confirm those of the first two studies, suggesting some benefits of expansive-open postures on divergent thinking; by inducing a momentary feeling of power, expansive postures may have led participants to process information holistically (as opposed to analytically), improving their creative performance in a task requiring divergent thinking. 
To date, all the tasks used to measure creativity required divergent thinking to be solved. The present findings are consistent with previous studies demonstrating a positive effect of expansive postures on divergent thinking, measured verbally while participants perform the tasks (Andolfi et al., 2017; Hao et al., 2017). In the present studies, verbal and figural divergent thinking were measured in written form after participants had adopted expansive postures. As far as we know, the benefits of postures have never been examined on creativity tasks requiring convergent thinking processes. This was the purpose of the fourth study, which investigated whether the effect of postures extended to closed-ended creativity tasks requiring convergent thinking. One of the most widely used measures of convergent creative thinking is the Remote Associates Test (RAT, Mednick, 1962). Although the RAT as a measure of creativity is debatable (Lubart, 2016), when examining its psychometric properties, Lee, Huggins and Therriault (2014) found that it assesses similar cognitive processes to those of other convergent thinking tests, and can be clearly distinguished from traditional divergent thinking tests of creativity. It comes within the category of insight problems, commonly used to assess creative problem solving. According to Guilford's (1950) definition of different types of creativity tasks, insight problems involve convergent thinking, in that each problem has only one correct solution (Metcalfe \& Wiebe, 1987). As the problems are ill defined, it is not immediately obvious how to solve them, but finding the solution is generally associated with an "aha!" or "flash of insight" experience. It may be the result of cognitive restructuring, a sudden change in the way the problem is perceived (Weisberg, 1995), and/or an associative process leading the solver to move from one idea to another through a chain of associations in memory (Durso, Rea, \& Dayton, 1994). It was expected that participants who have been placed in contractive rather than expansive postures would process information analytically and in detail, leading to better creative performance 
on creativity tasks requiring convergent thinking to find the correct solutions to a series of insight problems.

\section{Study 4: Effects of Postures on the Remote Associates Test}

\section{Method}

Participants and overview of the procedure

Eighty-two participants (61 females and 21 males), aged 18 to 31 years $(M=20.44$, $S D=2.50$ ), were randomly assigned to either an expansive or a contractive posture condition by a research assistant (one of the two female research assistants). A Chi-square test revealed no significant difference in the distribution of males and females between the two conditions, $\chi^{2}(1, N=82)=1.600, p=.206, \phi=.140$. As in the previous studies, all the participants were students in various disciplines, the majority in psychology $(N=26)$, arts and languages $(N=$ 17) and humanities $(N=9)$, and the remainder in art and architecture $(N=8)$, law, administration, accounting, communication, economy $(N=7)$, health, social work and nursing $(N=5)$, learning sciences $(N=5), \operatorname{STEM}(N=3)$, and sports science $(N=2)$.

A similar procedure was used in this study, except that participants had to perform a verbal creativity task based on the Remote Associates Test (RAT, Mednick, 1962; Mednick \& Mednick, 1967). This task is used as a measure of convergent thinking to assess the creative potential of individuals from their production of a single, correct solution to a creative problem (Lee et al., 2014; Taft \& Rossiter, 1966). In this task, a series of three unrelated words are presented, and participants must find one word that can be associated with all three by means of synonymy, formation of a compound word, and/or semantic association. For example, the words CREAM/SKATE/WATER are associated with ICE by means of synonymy $($ ice $=$ water), formation of a compound word (ice-cream), and semantic 
association (ice skate). As creativity requires the ability to discover relationships between notions that have nothing to do with each other, creative convergent thinking is required to find a valid solution because the first and most obvious solution to the task is often not the correct one, so that more remote connections need to be retrieved in order to relate the three words to each other (Schooler et al., 1993).

The original version of the RAT contains 30 items, and the materials generally used in the task involve associations of English words that are part of the US culture. To our knowledge, there is no currently available French version, so we adapted and pretested the material for the present study. Based on a list of RAT items extracted from previous studies (e.g. Akbari Chermahini, Hickendorff, \& Hommel, 2012; Bowden \& Beeman, 2003; Lee et al., 2014), we created a set of 60 items of various degrees of difficulty with French words that can be associated through synonymy, formation of a compound word, or semantic association. Participants who did not participate in the experiment had to solve each item as quickly as possible, with no time limit. We then produced a set of 38 items ranging from very easy ( $100 \%$ of correct answers) to very difficult (5.9\% of correct answers): 15 easy items ( 70 $100 \%$ of correct items), 15 medium items (20-70\% of correct items), and 8 difficult items (5$20 \%$ of correct items). The percentage of correct answers for the complete set was $52.9 \%$ (see N. Michinov \& E. Michinov, 2020 for the Online Supplementary Material 3 for the replicability of the experiment).

Participants were given the list of items, ranging from the easiest to the most difficult, and were instructed to find a solution for as many as possible in 15 minutes. They were told that they could skip one or several items when they could not find the solution. When they had completed the task, the research assistant gave out the same questionnaire as in the other studies. After completing the questionnaire, participants were debriefed and thanked. 


\section{Measures of creative performance}

Three indicators were used to measure creative performance. The first was the number of completed items; the second was the number of correctly solved items; the third was the number of correct items divided by the number of completed items (efficiency).

\section{Results and discussion}

As in the other studies, statistical analyses to check the efficacy of the experimental manipulation indicated that 74 participants out of $82(90.2 \%)$ correctly recognized the four postures they had adopted, with exactly the same proportion in the two conditions, $\chi^{2}(1, N=$ $82)=0.000, p=1.000, \phi=.000$.

As predicted, a one-tailed test demonstrated that the number of correct items was higher in the contractive posture condition $(M=18.634$ and $S D=5.305)$ than in the expansive posture condition $(M=15.098$ and $S D=4.888$; mean difference $=3.537,95 \%$ confidence interval, or CI $=[1.662,+\infty]), t(80)=3.139, p=.001$, Cohen's $d=0.693$. A similar pattern was observed for efficiency (number of correct items / number of completed items), with higher scores in the contractive posture condition $(M=0.775$ and $S D=0.176)$ than in the expansive posture condition $(M=0.674$ and $S D=0.225$; mean difference $=0.101,95 \%$ confidence interval, or $\mathrm{CI}=[0.045,+\infty]), t(80)=2.260, p=.013$, Cohen's $d=0.499$. However, no significant difference was observed in the number of completed items, which was similar in the two conditions: contractive $(M=24.854$ and $S D=6.632)$ and expansive $(M=$ 23.585 and $S D=7.043$; mean difference $=1.268,95 \%$ confidence interval, or $\mathrm{CI}=[-1.246$, $+\infty]), t(80)=0.840, p=.404$, Cohen's $d=0.185^{3}$.

The results of this fourth study revealed, as predicted, that participants adopting contractive-closed postures had better creative performance on a task requiring convergent 
thinking than participants adopting expansive postures. These findings are important because they provide the first empirical demonstration of the benefits of contractive postures on behavior, and more specifically in creativity tasks involving convergent thinking. Previous studies have found a positive effect of expansive postures on creative performance, but only on tasks requiring divergent thinking. As suggested recently by other researchers (Andolfi et al., 2017; Hao et al., 2017), it is crucial to use convergent thinking tasks to test the generality of the effects of postural feedback on creativity. However, contractive postures have never been recognized as contributing to better creative performance than expansive postures, and they have essentially been used as a control condition in the literature.

In this study, we could expect to observe a reverse effect of contractive postures on creative performance for tasks requiring convergent thinking. This hypothesis was only confirmed with regard to the number of correct items and the efficiency measure (number of correct items / number of completed items); there was no significant difference in the number of completed items. In contrast to the results of our first three studies, it is possible that participants gave priority to quality over quantity after adopting contractive postures. In such postures, participants used a narrow (or local) type of information processing, examining different and unexpected angles to find the correct solution to the problem rather than completing a maximum of items. To extend the findings of this fourth study, another study was conducted in order to test the potential benefits of contractive postures on a series of other insight problems. As far as we know, Study 4 is the only one revealing a positive impact of contractive postures on creative performance in a task requiring convergent thinking, and these findings therefore need to be extended to similar creativity tasks. This is important in that there is an ongoing debate about what the RAT really measures. For some researchers (Lee et al. 2014), the RAT assesses cognitive processes similar to those involved in a wide range of other analytical and convergent thinking tests, clearly distinguishing it from 
divergent thinking tests of creativity. For other researchers (Lubart, 2016), the RAT is very similar to concepts of classic intelligence, and its use as a convergent thinking test for creativity is debatable. Consequently, we tested our hypothesis using another (verbal) convergent thinking task based on a different series of insight problems.

\section{Study 5: Effects of Postures on insight problems}

\section{Method}

Participants and overview of the procedure

Eighty-four participants (56 females and 28 males), aged 18 to 33 years $(M=21.08$, $S D=2.39$ ), were randomly assigned to either the expansive or contractive posture condition (28 females and 14 males per condition). A Chi-square test revealed no significant difference in the distribution of males and females between the two conditions, $\chi^{2}(1, N=84)=0.000, p$ $=1.00, \phi=0.00$. As in previous studies, all the participants were students in various disciplines, the majority in psychology $(N=36)$, art and architecture $(N=12)$, law, administration, accounting, communication, economy $(N=12)$, and humanities $(N=9)$. The rest were in arts and languages $(N=7)$, learning sciences $(N=4), \operatorname{STEM}(N=3)$, and sports science $(N=1)$.

A similar procedure was used in this study, except that participants carried out a convergent thinking task based on a series of insight problems (Webb, Little, \& Cropper, 2016), pretested to determine their difficulty level. In the pretest, 40 participants (28 females and 12 males), all university students in various disciplines, had to solve 17 verbal insight problems. The problems were taken from other studies and translated into French (e.g., Beaty et al., 2014; DeYoung, Flanders, \& Peterson, 2008; Dow \& Mayer, 2004). They were presented in a booklet, and participants were given forty-five minutes to solve them. The 
proportion of correct answers was calculated for each problem. Based on these rates, and to be comparable to the procedure of Study 4 where items were organized from the easiest to the most difficult, 6 problems were selected: two easy $(>70 \%$ of correct solutions, pear tree and ancient invention), two difficult $(<25 \%$, shifting gears and archeologist), and two of medium difficulty (around 30-40\%, fishing and light switch) (see N. Michinov \& E. Michinov, 2020 for the Online Supplementary Material 4).

After completing and signing a consent form, participants were assigned to either the expansive or the contractive posture condition by a research assistant. The problems were presented in a booklet in order of difficulty, and participants were told that they had 15 minutes to solve the six problems and that they should go to the next problem if they could not find the solution to a problem. The instructions were presented on a piece of paper placed upside down on the table.

At the end of the task, the experimenter came into the room and gave the participant the same questionnaire as in the other studies as well as two measures of feeling of power and familiarity with Amy Cuddy’s TED talk (2012) ${ }^{4}$. Finally, participants were debriefed and thanked.

Measures of creative performance

Three indicators were used to measure creative performance. The first was the number of completed problems; the second was the number of correctly solved problems; the third was the number of correctly solved problems accordingly to their degree of difficulty (easy, medium or difficult).

\section{Results and discussion}


Statistical analyses to check the efficacy of the experimental manipulation indicated that 80 participants out of $84(95.2 \%)$ correctly recognized the four postures they had adopted, with exactly the same proportion in both conditions, $\chi^{2}(1, N=84)=1.050, p=.306, \phi=$ .112.

As the data were not normally distributed (the Shapiro-Wilk test is significant at $p<$ .001), a Wilcoxon rank-sum test was performed. It revealed that the number of solved problems was not significantly higher in the contractive posture condition $(M d n=6.00)$ than in the expansive posture condition $(M d n=6.00), W_{s}=869.5, p=0.824, r_{B}=-0.082$. It is important to note that a similar and very high proportion of participants attempted to solve four to six problems $(95.2 \%$ and $100 \%$ in the contractive and expansive posture conditions, respectively). Contrary to our predictions, the number of correctly solved problems was not significantly higher in the contractive posture condition $(M d n=3.00)$ than in the expansive posture condition $(M d n=2.50), W_{s}=871.5, p=0.540, r_{B}=-0.012$. Descriptive results show that nearly $25 \%$ of participants correctly solved two or three problems $(23.8 \%$ and $21.4 \%$, respectively), with lower rates for the other possibilities $(1$ problem $=16.7 \% ; 4$ problems $=$ $14.3 \% ; 5$ problems $=10.7 \% ; 0$ problem $=8.3 \% ; 6$ problems $=4.8 \%)$. Analyses of each category of problems revealed that the two easy problems were correctly solved more in the contractive posture condition $(M d n=2.00)$ than in the expansive posture condition $(M d n=$ 1.00), $W_{s}=1059.0, p=.040, r_{B}=0.201$. There was no difference for the two medium problems $(M d n=1.00$ and $M d n=1.00$, respectively $), W_{s}=817.0, p=.735, r_{B}=-0.074$, or the two difficult problems $\left(M d n=0.00\right.$ and $M d n=0.00$, respectively), $W_{s}=774.5, p=.870, r_{B}=$ - 0.122. Further analyses were performed on the number of participants who found 0 to 2 correct answers for the two easy, medium, or difficult problems. Results revealed a different proportion of participants who found the correct answers for the two easy problems $(61.9 \%$ in the contractive posture condition and $38.1 \%$ in the expansive posture condition), $\chi^{2}(2, N=$ 
$84)=6.159, p=.046, \phi=.271$. No difference was found for the medium and difficult problems (see Table 1).

Table 1. Percentage of 0 to 2 correct answers to the insight problems (easy, medium, difficult) per experimental condition

\begin{tabular}{ccccccc}
\hline $\begin{array}{c}\text { Correct } \\
\text { answers }\end{array}$ & \multicolumn{3}{c}{ Expansive postures } & \multicolumn{3}{c}{ Contractive postures } \\
\hline & Easy & Medium & Difficult & Easy & Medium & Difficult \\
\hline 0 & 9.5 & 42.9 & 54.8 & 11.9 & 47.6 & 69.0 \\
\hline 1 & 52.4 & 26.2 & 38.1 & 26.2 & 28.6 & 21.4 \\
\hline 2 & 38.1 & 31.0 & 7.1 & 61.9 & 23.8 & 9.5 \\
\hline
\end{tabular}

The aim of this fifth study was to extend the results of Study 4 and examine whether adopting contractive-closed postures would lead to better creative performance than expansive-open postures on verbal insight tasks requiring convergent thinking. The findings partially replicated those of the fourth study, but the difference between the two conditions was only observed for the two easy problems, which were correctly solved by nearly twice as many participants in contractive-closed postures than in expansive-open postures $(61.9 \%$ and $38.1 \%$, respectively). However, we failed to demonstrate a difference between contractive and expansive posture conditions on the insight problems as a whole, independently of their degree of difficulty. While participants tried to solve almost all the problems in the 15 minutes, they were likely to have started with the first (and easiest) problems, i.e. immediately after adopting the postures. It is therefore possible that the effect of the posture was strongest when solving those problems.

Surprisingly, the effect of expansive postures on the feeling of power measured by post-experimental questionnaire was not found in this study, contrary to the findings of Cuddy and collaborators' (2018) review, and of studies measuring this feeling immediately after 
performing a gambling task (see Bombari et al., 2017). It is possible that the absence of effect was due to the time between the postures and completing the questionnaire. This interval was probably very short for a gambling task, compared to the current studies where the questionnaire was administered 15 minutes after participants had been placed in four distinct postures. The effect of postures on emotions, attitudes, evaluations, and probably many other behaviors is likely to be short-lived, but currently we do not know the duration of the effects of posture on different outcomes. The lapse of time between the posture manipulations and participants' behaviors requires greater examination in future studies (see Cuddy et al., 2018). A summary of results for the five studies is presented in the Table 2. 
Table 2. Summary of Means (and Standard Deviations) on Each Creative Performance Measure for the Five Studies in Function of the Condition of Postures (Expansive versus Contractive).

\begin{tabular}{|c|c|c|c|c|c|c|c|}
\hline \multirow{2}{*}{ Studies/Tasks/Measures } & & \multicolumn{2}{|c|}{ Expansive postures } & \multicolumn{2}{|c|}{ Contractive postures } & \multirow[t]{2}{*}{ Sig. } & \multirow[t]{2}{*}{$d$ of Cohen } \\
\hline & & $\mathrm{M}$ & SD & $\mathrm{M}$ & SD & & \\
\hline \multirow{3}{*}{$\begin{array}{l}\text { Study } 1(N=76) \text {. Idea- } \\
\text { generation task (Verbal } \\
\text { divergent thinking) }\end{array}$} & Fluency & 15.97 & 7.35 & 12.10 & 4.51 & .004 & 0.634 \\
\hline & Flexibility & 5.55 & 1.48 & 5.05 & 1.41 & .068 & 0.340 \\
\hline & Originality & 1.95 & 0.33 & 1.89 & 0.30 & .24 & 0.160 \\
\hline $\begin{array}{l}\text { Study } 2(N=70) . \text { Creative } \\
\text { Invention Task (Figural } \\
\text { divergent thinking) }\end{array}$ & Originality (sort-resort technique) & 4.68 & 1.50 & 3.60 & 1.46 & .002 & 0.728 \\
\hline \multirow{4}{*}{$\begin{array}{l}\text { Study } 3(N=44) \text {. Drawing } \\
\text { production task (Figural } \\
\text { divergent thinking) }\end{array}$} & Overall creativity & 28.30 & 8.46 & 23.92 & 7.08 & .035 & 0.561 \\
\hline & Conventionality scores & 25.79 & 8.05 & 21.60 & 6.34 & .031 & 0.579 \\
\hline & Unconventionality scores & 2.515 & 1.59 & 2.333 & 1.95 & .368 & 0.102 \\
\hline & Originality (sort-resort technique) & 5.16 & 1.64 & 3.73 & 2.08 & .008 & 0.764 \\
\hline \multirow{3}{*}{$\begin{array}{l}\text { Study } 4(N=82) \text {. Remote } \\
\text { Association Task (Verbal } \\
\text { convergent thinking) }\end{array}$} & Number of total items & 23.58 & 7.04 & 24.85 & 6.63 & .40 & 0.185 \\
\hline & Number of correct items & 15.09 & 4.89 & 18.63 & 5.30 & .001 & 0.693 \\
\hline & Efficiency (correct/total) & 0.674 & 0.22 & 0.77 & 0.17 & .013 & 0.499 \\
\hline \multirow{6}{*}{$\begin{array}{l}\text { Study } 5(N=84) \text {. Insight } \\
\text { problems (Verbal convergent } \\
\text { thinking) }\end{array}$} & Number of completed problems & 5.786 & 0.520 & 5.595 & 0.828 & .82 & -- \\
\hline & Number of correctly solved problems & 2.690 & 1.506 & 2.667 & 1.720 & .54 & -- \\
\hline & Difficulty of correctly solved problems & & & & & & \\
\hline & Easy & 1.286 & 0.636 & 1.500 & 0.707 & .040 & -- \\
\hline & Medium & 0.881 & 0.861 & 0.762 & 0.821 & .73 & -- \\
\hline & Difficult & 0.524 & 0.634 & 0.405 & 0.665 & .87 & -- \\
\hline
\end{tabular}




\section{General discussion}

The present research aimed to examine whether expansive postures have a positive effect on divergent creative thinking, and whether contractive postures have a positive effect on convergent creative thinking. Five experiments investigated these predictions using a series of tasks involving divergent and convergent thinking processes. First, in line with previous results (Andolfi et al., 2017; Hao et al., 2017), we demonstrated the positive effect of expansive postures on creativity tasks involving divergent thinking processes. Secondly, new findings were obtained in the last two studies, demonstrating the reverse effect: contractive postures had a positive effect on creativity tasks involving convergent thinking processes.

These findings extend research about the effects of postural feedback, suggesting that contractive postures may also have positive psychological and behavioral effects, and should not be considered only as a control condition. Indeed, contractive postures may also have an impact on performance on creativity tasks requiring convergent thinking processes in order to find the single correct solution to a problem. Thus, the present findings show that the benefits of expansive postures on creative performance appeared for divergent thinking tasks. Conversely, the benefits of contractive postures appeared for convergent thinking tasks. Taken together, the results of the present studies suggest that expansive postures led the participants to process information holistically in order to explore a large number of ideas or solutions in divergent thinking tasks. Thus, participants who had been momentarily placed in expansive postures (presumed to create a strong feeling of power) performed better in creativity tasks requiring divergent thinking. Conversely, contractive postures led participants to process information analytically, increasing attentional focus on tasks in order to examine different and unexpected angles of a problem to "discover" the correct solution. Thus, participants who had been momentarily placed in contractive postures (presumed to create a 
weak feeling of power) performed better in creativity tasks requiring convergent thinking. These findings are consistent with research using different experimental procedures to induce a feeling of power (e.g., Galinsky et al., 2008; Sligte et al., 2011; Smith \& Trope, 2006). However, the lack of cognitive measures during the task, or immediately after the posture phase, prevented us from verifying whether participants had used a holistic/global or an analytical/local mode of information processing. These two processes were only inferred from creative performance on two categories of tasks requiring either divergent or convergent thinking.

In addition to an interpretation based on different types of information processing, other mediating processes may be at work to influence the impact of posture on creative thinking, such as feeling of power, perception of the situation, and emotion activation. First, previous findings have demonstrated that expansive postures increase the feeling of power (Bombari et al., 2017; Cuddy et al., 2018; Gronau et al., 2017), and that inducing a feeling of power by postural openness may have a positive impact on creative performance on tasks requiring divergent thinking. However, this does not explain the reverse effect observed in contractive postures, which were found to have a positive influence on creative performance on tasks requiring convergent thinking. Secondly, it is possible that specific postures affect the way participants perceive the situation, which may explain the results found in these studies. Indeed, after holding expansive postures, participants may perceive a relaxed atmosphere, leading them to "liberate" their creativity, at least when the tasks require divergent thinking. This interpretation is consistent with findings that disorderly environments have a stimulating effect on divergent thinking tasks, compared to orderly environments (Vohs, Redden, \& Rahinel, 2013). As expansive postures may generate a relaxed atmosphere similar to that generally observed in disorderly environments, they may benefit divergent thinking. Conversely, contractive postures may be perceived as generating a tense 
atmosphere, as in orderly environments, which may explain the positive effect on creativity tasks requiring convergent thinking. Thirdly, the activation of different emotions by specific postures may also explain creative performance (Riskind \& Gotay, 1982). On the one hand, it has been demonstrated that expansive-open postures lead to more positive emotions, and that contractive-closed postures produce more negative emotions (Rossberg-Gempton \& Poole, 1992). On the other hand, it has been recognized that divergent thinking leads to positive emotions whereas convergent thinking leads to negative emotions (Chermahini \& Hommel, 2012). These findings suggest that emotion activation may mediate the impact of postures on creative thinking. Although a previous study failed to demonstrate any difference between expansive and contractive postures on emotional activation by means of several physiological indicators (Andolfi et al., 2017, Study 2), future research should address the question of how and when to measure cognitive and/or emotional processes without being intrusive during the task. More broadly, and based on Darwin's (1872) evolutionary approach, it has been suggested that different postures may evoke different emotions, and that expansive and contractive postures are ancient signals used by different animal species to express emotions (see Metzler, 2018). From this perspective, emotions are considered as predispositions to act adaptively, and specific body movements are associated with emotional states and their neuronal correlates. More specifically, mirror neurons (a type of cortical neuron involved in emotions) are activated when an individual performs an action or observes an individual performing an action, thereby preparing the brain for action (de Gelder, Snyder, Greve, Gerard, \& Hadjikhani, 2004). Thus, adopting some postures may have produced activity in brain areas narrowly associated with emotions, and this emotion-related activity may have influenced creative thinking processes. Future studies are needed to verify this hypothesis.

In addition to these alternative interpretations, our findings contribute to a better understanding of the scientific controversy about the effect (or absence of effect) of expansive 
postures on various psychological outcomes. Indeed, statistical arguments based on a p-curve analysis indicate that the effects of expansive postures are less convincing for non-EASE variables, including creativity (Cuddy et al., 2018). Moreover, most of the studies using creativity tasks involved divergent thinking processes and found positive effects of expansive postures (see Malinin, 2019). However, mixed results were found when non-creative tasks were used. For example, Huang and collaborators (2011) found that when participants were shown images of incomplete pictures and asked to identify them, those in the expansive posture condition correctly identified more pictures than those in the contractive posture condition. However, Cesario and Johnson (2017) failed to replicate this effect. Similarly, Andolfi and collaborators (2017) found no significant difference between the expansive and contractive postures on a non-creative logical reasoning task in which participants had to solve a series of syllogisms. These findings highlight the difficulty of generalizing the effects of postural feedback to cognitive tasks other than creativity tasks, and additional research is needed to better understand the effect of postures on cognitive task performance. Future research should also extend the classic two-step paradigm used in the postural feedback literature. One way to proceed would be to conduct written verbal and figural creativity tasks (requiring either divergent or convergent thinking) while participants adopt expansive or contractive postures.

\section{Conclusion}

This series of studies adds to the debate about the controversial effect of nonverbal physical postures expressing power on psychological and behavioral outcomes. They not only confirmed the positive effect of expansive postures on creative divergent thinking, but they also identified the benefits of contractive postures on convergent thinking. Thus, creative performance on tasks requiring different creative thinking processes was affected by different 
postures. In conclusion, this research offers new perspectives for embodied creativity research (Frith et al., 2019; Malinin, 2019; Stanciu, 2015), suggesting that the body may have an influence on creative thinking, depending on the type of posture and creativity task to be performed. This could lead to new views about how the body shapes a creative mindset. 


\section{References}

Akbari Chermahini, S. A., Hickendorff, M., \& Hommel, B. (2012). Development and validity of a Dutch version of the Remote Associates Task: An item-response theory approach. Thinking Skills and Creativity, 7, 177-186. https://doi.org/10.1016/j.tsc.2012.02.003

Akbari Chermahini, S. A., \& Hommel, B. (2012). Creative mood swings: Divergent and convergent thinking affect mood in opposite ways. Psychological Research, 76, 634640. https://doi.org/10.1007/s00426-011-0358-z

Amabile, T. M. (1982). Social psychology of creativity: A consensual assessment technique. Journal of Personality and Social Psychology, 43(5), 997-1013. https://doi.org/10.1037/0022-3514.43.5.997

Andolfi, V. R., Di Nuzzo, C., \& Antonietti, A. (2017). Opening the mind through the body: The effects of posture on creative processes. Thinking Skills and Creativity, 24, 20-28. https://doi.org/10.1016/j.tsc.2017.02.012

Bailey, A. H., LaFrance, M., \& Dovidio, J. F. (2017). Could a woman be superman? Gender and the embodiment of power postures. Comprehensive Results in Social Psychology. https://doi.org/10.1080/23743603.2016.1248079

Barsalou, L. W. (2008). Grounded cognition. Annual Review of Psychology, 59, 617-645. https://doi.org/10.1146/annurev.psych.59.103006.093639

Beaty, R. E., Nusbaum, E. C., \& Silvia, P. J. (2014). Does insight problem solving predict real-world creativity? Psychology of Aesthetics, Creativity, and the Arts, 8(3), 287292. https://doi.org/10.1037/a0035727 
Beersma, B., \& De Dreu, C. K. W. (2005). Conflict's consequences: Effects of social motives on postnegotiation creative and convergent group functioning and performance. Journal of Personality and Social Psychology, 89(3), 358-374. https://doi.org/10.1037/0022-3514.89.3.358

Bohns, V. K., \& Wiltermuth, S. S. (2012). It hurts when I do this (or you do that): Posture and pain tolerance. Journal of Experimental Social Psychology, 48(1), 341-345. https://doi.org/10.1016/j.jesp.2011.05.022

Bombari, D., Schmid Mast, M., \& Pulfrey, C. (2017). Real and imagined power poses: Is the physical experience necessary after all? Comprehensive Results in Social Psychology, 1, 44-54. https://doi.org/10.1080/23743603.2017.1341183

Bowden, E. M., \& Beeman, M. J. (2003). Normative data for 144 compound remote associate problems. Behavior Research Methods, Instruments, and Computers, 35, 634-639. https://doi.org/10.3758/BF03195543

Carney, D. R., Cuddy, A. J. C., \& Yap, A. J. (2010). Power posing: Brief nonverbal displays affect neuroendocrine levels and risk tolerance. Psychological Science, 21(10), 13631368. https://doi.org/10.1177/0956797610383437

Carney, D. R., Cuddy, A. J. C., \& Yap, A. J. (2015). Review and summary of research on the embodied effects of expansive (vs. contractive) nonverbal displays. Psychological Science, 1-7. https://doi.org/10.1177/0956797614566855

Cesario, J., \& McDonald, M. M. (2013). Bodies in context: Power poses as a computation of action possibility. Social Cognition, 31(2), 260-274. https://doi.org/10.1521/soco.2013.31.2.260 
Cesario, J, \& Johnson, D. J. (2017). Power poseur: Bodily expansiveness does not matter in dyadic interactions. Social Psychological and Personality Science, 1-9. https://doi.org/ $10.1177 / 1948550617725153$

Cesario, J., Jonas, K. J., \& Carney, D. R. (2017). CRSP special issue on power poses: what was the point and what did we learn? Comprehensive Results in Social Psychology, 2, 1-5. https://doi.org/10.1080/23743603.2017.1309876

Craig, T. Y., \& Kelly, J. R. (1999). Group cohesiveness and creative performance. Group Dynamics: Theory, Research, and Practice, 3, 243-256. https://doi.org/10.1037/10892699.3.4.243

Credé, M., \& Phillips, L. A. (2017). Revisiting the power pose effect: How robust are the results reported by Carney, Cuddy, and Yap (2010) to data analytic decisions? Social Psychological and Personality Science, 8(5), 493-499. https://doi.org/ $10.1177 / 1948550617714584$

Cuddy, A. J. C. (2012). Your body language shapes who you are. Retrieved from http://www.ted.com/talks/amy_cuddy_your_body_language_shapes_who_you_are?c= 913722

Cuddy, A. J. C. (2015). Presence: Bringing your boldest self to your biggest challenges. New York: Little, Brown and Company.

Cuddy, A. J. C., Schultz, S. J., \& Fosse, N. E. (2018). P-curving a more comprehensive body of research on postural feedback reveals clear evidential value for "Power Posing" effects: Reply to Simmons and Simonsohn. Psychological Science, 1-11. https://doi.org/10.1177/0956797617746749 
Cuddy, A. J. C., Wilmuth, C. A., Yap, A. J., \& Carney, D. R. (2015). Preparatory power posing affects nonverbal presence and job interview performance. Journal of Applied Psychology, 100, 1286-1295. https://doi.org/10.1037/a0038543

Darwin, C. R. (1872). The expression of the emotions in man and animals. London: John Murray.

de Gelder, B., Snyder, J., Greve, D., Gerard, G., \& Hadjikhani, N. (2004). Fear fosters flight: A mechanism for fear contagion when perceiving emotion expressed by a whole body. Proceedings of the National Academy of Sciences of the United States of America, 101(47), 16701. https://doi.org/10.1073/pnas.0407042101

DeYoung, C. G., Flanders, J. L., \& Peterson, J. B. (2008). Cognitive abilities involved in insight problem solving: An individual differences model. Creativity Research Journal, 20(3), 278-290. https://doi.org/10.1080/10400410802278719

Dow, G.T. \& Mayer, R.E. (2004). Teaching students to solve insight problems. Evidence for domain specificity in training. Creativity Research Journal, 16,4 389-402. https://doi.org/10.1080/10400410409534550

Duncker, K. (1945). On problem-solving (L. S. Lees, Trans.). Psychological Monographs, 58(5), i-113. https://doi.org/10.1037/h0093599

Durso, F. T., Rea, C. B., \& Dayton, T. (1994). Graph-theoretic confirmation of restructuring during insight. Psychological Science, 5(2), 94-98. https://doi.org/10.1111/j.14679280.1994.tb00637.x 
Faul, F., Erdfelder, E., Buchner, A., \& Lang, A.-G. (2009). Statistical power analyses using G*Power 3.1: Tests for correlation and regression analyses. Behavior Research Methods, 41, 1149-1160. https://doi.org/10.3758/BRM.41.4.1149

Finke, R. A. (1990). Creative imagery: Discoveries and inventions in visualization. Hillsdale, NJ: Erlbaum.

Finke, R. A., Ward, T. B., \& Smith, S. M. (1992). Creative cognition: Theory, research, and applications. Cambridge MA: MIT Press.

Friedman, R. S., Fishbach, A., Förster, J., \& Werth, L. (2003). Attentional priming effects on creativity. Creativity Research Journal, 15, 277-286. https://doi.org/10.1080/10400419.2003.9651420

Friedman, R. S., \& Förster, J. (2000). The effects of approach and avoidance motor actions on the elements of creative insight. Journal of Personality and Social Psychology, 79, 477-492. https://doi.org/10.1037/0022-3514.79.4.477

Friedman, R. S., \& Förster, J. (2002). The influence of approach and avoidance motor actions on creative cognition. Journal of Experimental Social Psychology, 38(1), 41-55. https://doi.org/10.1006/jesp.2001.1488

Frith, E., Miller, S., \& Loprinzi, P. D. (2019). A Review of experimental research on embodied creativity: Revisiting the mind-body connection. The Journal of Creative Behavior. https://doi.org/10.1002/jocb.406

Galinsky, A. D., Magee, J. C., Gruenfeld, H. G., Whitson, J. A., \& Liljenquist, K. A. (2008). Power reduces the press of the situation: Implications for creativity, conformity, and 
dissonance. Journal of Personality and Social Psychology, 95, 1450-1466. https://doi.org/10.1037/a0012633

Galinsky, A. D., Rucker, D. D., \& Magee, J. C. (2015). Power: Past findings, present considerations, and future directions. In M. Mikulincer, P. R. Shaver, J. A. Simpson, \& J. F. Dovidio (Eds.), APA handbooks in psychology ${ }^{\circledR}$. APA handbook of personality and social psychology, Vol. 3. Interpersonal relations (pp. 421-460). American Psychological Association. https://doi.org/10.1037/14344-016

Gervais, S. J., Guinote, A., Allen, J., \& Slabu, L. (2013). Power increases situated creativity. Social Influence, 8(4), 294-311. https://doi.org/10.1080/15534510.2012

Guinote, A. (2007). Power affects basic cognition: Increased attentional inhibition and flexibility. Journal of Experimental Social Psychology, 43, 685-697. https://doi.org/10.1016/j.jesp.2006.06.008

Gronau, Q. F., Van Erp, S., Heck, D. W., Cesario, J., Jonas, K. J., \& Wagenmakers, E.-J. (2017). A Bayesian model averaged meta-analysis of the power pose effect with informed and default priors: The case of felt power. Comprehensive Results in Social Psychology, 2, 123-138. https://doi.org/10.1080/23743603.2017.1326760

Guilford, J. P. (1950). Creativity. American Psychologist, 5(9), 444-454. https://doi.org/10.1037/h0063487

Guilford, J. P. (1967). Creativity: yesterday, today, and tomorrow. The Journal of Creative Behavior, 1, 3-14. https://doi.org/10.1002/j.2162-6057.1967.tb00002.x 
Hackman, J. R., Jones, L. E., \& McGrath, J. E. (1967). A set of dimensions for describing the general properties of group-generated written passages. Psychological Bulletin, 67, 379-390. https://doi.org/10.1037/h0024647

Hall, J. A., Coats, E. J., \& LeBeau, L. S. (2005). Nonverbal behaviour and the vertical dimension of social relations: A meta-analysis. Psychological Bulletin, 131(6), 898924. https://doi.org/10.1037/0033-2909.131.6.898

Hao, N., Xue, H., Yuan, H., Wang, Q., \& Runco, M. A. (2017). Enhancing creativity: Proper body posture meets proper emotion. Acta Psychologica, 173, 32-40. https://doi.org/10.1016/j.actpsy.2016.12.005

Huang, L., Galinsky, A. D., Gruenfeld, D. H., \& Guillory, L. E. (2011). Powerful postures versus powerful roles: Which is the proximate correlate of thought and behavior? Psychological Science, 22(1), 95-102. https://doi.org/10.1177/0956797610391912

James, W. (1894). Discussion: The physical basis of emotion. Psychological Review, 1, 516529. https://doi.org/10.1037/h0065078

Jellen, H. G., \& Urban, K. K. (1989). Assessing creative potential world-wide: The first crosscultural application of the test for creative thinking drawing production (TCT-DP). Gifted Education International, 6, 78-86. https://doi.org/10.1177/026142948900600204

Jonas, K., Cesario, J., Alger, M., Bailey, A. H., Bombari, D., Carney, D., Dovidio, J. F., Duffy, S., Harder, J., van Huistee, D., Jackson, B., Johnson, D. J., Keller, V. N., Klaschinski, L., LaBelle, O., LaFrance, M., Latu, I. M., Morssinkhoff, M., Nault, K., 
Pardal, V., Pulfrey, C., Rohleder, N., Ronay, R., Smart Richman, L., Schmid Mast, M., Schnabel, K., Schröder-Abé, M., \& Tybur, J. M. (2017). Power poses - where do we stand? Comprehensive Results in Social Psychology, 2, 139-141. https://doi.org/10.1080/23743603.2017.1342447

Jones, E. E., \& Kelly, J. R. (2009). No pain, no gains: Negative mood leads to process gains in idea-generation groups. Group Dynamics: Theory, Research, and Practice, 13(2), 75-88. https://doi.org/10.1037/a0013812

Kelly, J. R., \& Karau, S. J. (1993). Entrainment of creativity in small groups. Small Group Research, 24(2), 179-198. https://doi.org/10.1177/1046496493242002

Keltner, D., Gruenfeld, D., \& Anderson, C. (2003). Power, approach, and inhibition. Psychological Review, 110, 265-284. https://doi.org/10.1037/0033-295X.110.2.265

Körner, R., Petersen, L.-E., \& Schütz, A. J. C. P. (2019). Do expansive or contractive body postures affect feelings of self-worth? High power poses impact state self-esteem. Current Psychology. https://doi.org/10.1007/s12144-019-00371-1

Latu, I. M., Schmid Mast, M., Bombari, D., Lammers, J. \& Hoyt, C. L. (2018). Empowering mimicry: Female leader role models empower women in leadership tasks through body posture mimicry. Sex Roles, 80(1-2), 11-24. https://doi.org/10.1007/s11199-0180911-y

Lee, C. S., Huggins, A. C., \& Therriault, D. J. (2014). A measure of creativity or intelligence? Examining internal and external structure validity evidence of the Remote Associates Test. Psychology of Aesthetics, Creativity, and the Arts, 8, 446-460. https://doi.org/10.1037/a0036773 
Lubart, T. (2016). Creativity and convergent thinking: Reflections, connections and practical considerations. RUDN Journal of Psychology and Pedagogics, 4, 7-15. https://doi.org/10.22363/2313-1683-2016-4-7-15

Maier, N. R. F. (1930). Reasoning in humans: I. On direction. Journal of Comparative Psychology, 10, 115-143. https://doi.org/10.1037/h0073232

Malinin, L. H. (2019). How radical is embodied creativity? Implications of 4E approaches for creativity research and teaching. Frontiers in Psychology, 10(2372). https://doi.org/10.3389/fpsyg.2019.02372

Mednick, S. (1962). The associative basis of the creative process. Psychological Review, 69(3), 220-232. https://doi.org/10.1037/h0048850

Mednick, S. A., \& Mednick, M. T. (1967). Examiner's manual, Remote Associates Test: College and adult forms 1 and 2. Boston: Houghton Mifflin.

Mehta, R., \& Dahl, D.W. (2019). Creativity: Past, present, and future. Consumer Psychology Review, 2, 30- 49. https://doi.org/10.1002/arcp.1044

Mehta, R., \& Zhu, R. J. (2009). Blue or red? Exploring the effect of color on cognitive task performance. Science, 323, 1226-1229. https://doi.org/10.1126/science.1169144

Meier, F. C., Schöbel, M., \& Feufel, M. A. (2019): Does size matter? Spacious car cockpits may increase the probability of parking violations, Ergonomics, 61(12), 1613-1618. https://doi.org/10.1080/00140139.2018.1503727

Metcalfe, J., \& Wiebe, D. (1987). Intuition in insight and noninsight problem solving. Memory and Cognition, 15(3), 238-246. https://doi.org/10.3758/BF03197722 
Metzler, H. (2018). The influence of bodily actions on social perception and behaviour: assessing effects of power postures. Unpublished doctoral Dissertation, Sorbonne Université. Available online at: https://www.theses.fr/2018SORUS287

Metzler, H., \& Grèzes, J. (2019). Repeatedly adopting power postures does not affect hormonal correlates of dominance and affiliative behavior. PeerJ, 7, e6726, https://doi.org/10.7717/peerj.6726

Michinov, N., Jamet, E., Métayer, N., \& Le Hénaff, B. (2015). The eyes of creativity: Impact of social comparison and individual creativity on performance and attention to others' idea during electronic brainstorming. Computers in Human Behavior, 42, 57-67. https://doi.org/10.1016/j.chb.2014.04.037

Michinov, N., \& Michinov, E. (2020, July 10). Postural feedback and creative thinking. Retrieved from osf.io/c9mzh

Niedenthal, P. M., Barsalou, L. W., Winkielman, P., Krauth-Gruber, S., \& Ric, F. (2005). Embodiment in attitudes, social perception, and emotion. Personality and Social Psychology Review, 9(3), 184-211. https://doi.org/10.1207/s15327957pspr0903_1

Oppezzo, M., \& Schwartz, D. L. (2014). Give your ideas some legs: The positive effect of walking on creative thinking. Journal of Experimental Psychology: Learning, Memory, and Cognition, 40(4), 1142. https://doi.org/10.1037/a0036577

Overbeck, J. R., \& Park, B. (2006). Powerful perceivers, powerless objects: Flexibility of powerholders' social attention. Organizational Behavior and Human Decision Processes, 99 (2), 227-243. https://doi.org/10.1016/j.obhdp.2005.10.003 
Park, L. E., Streamer, L., Huang, L., \& Galinsky, A. D. (2013). Stand tall, but don't put your feet up: Universal and culturally-specific effects of expansive postures on power. Journal of Experimental Social Psychology, 49, 965-971. https://doi.org/10.1016/j.jesp.2013.06.001

Perugini, M., Gallucci, M., \& Costantini, G. (2018). A practical primer to power analysis for simple experimental designs. International Review of Social Psychology, 31(1), 20. https://doi.org/10.5334/irsp.181

Ranehill, E., Dreber, A., Johannesson, M., Leiberg, S., Sul, S., \& Weber, R. A. (2015). Assessing the robustness of power posing: No effect on hormones and risk tolerance in a large sample of men and women. Psychological Science, 26, 653-656. https://doi.org/10.1177/0956797614553946

Riskind, J. H. (1984). They stoop to conquer - guiding and self-regulatory functions of physical posture after success and failure. Journal of Personality and Social Psychology, 47(3), 479-493. https://doi.org/10.1037/0022-3514.47.3.479

Riskind, J. H., \& Gotay, C. C. (1982). Physical posture: Could it have regulatory or feedback effects on motivation and emotion? Motivation and Emotion, 6(3), 273-298. https://doi.org/10.1007/BF00992249

Ronay, R., Tybur, J. M., van Huijstee, D., \& Morssinkhof, M. (2017). Embodied power, testosterone, and overconfidence as a causal pathway to risk-taking. Comprehensive Results in Social Psychology, 2(1), 28-43. https://doi.org/10.1080/23743603.2016.1248081 
Rossberg-Gempton, I., \& Poole, G. D. (1992). The relationship between body movement and affect: From historical and current perspectives. The Arts in Psychotherapy, 19(1), 3946. https://doi.org/10.1016/0197-4556(92)90062-S

Schooler, J. W., \& Melcher, J. (1995). The ineffability of insight. In S. M. Smith, M. Steven, T. B. Ward, \& R. A. Finke (Eds.), The Creative Cognition Approach (pp. 97-133). Cambridge, MA: The MIT Press.

Schooler, J. W., Ohlsson, S., \& Brooks, K. (1993). Thoughts beyond words: When language overshadows insight. Journal of Experimental Psychology: General, 122, 166-183. https://doi.org/10.1037/0096-3445.122.2.166

Shaw, M. E. (1963). Scaling group tasks: A method for dimensional analysis. Tech. Report No. 1, Contract NR 170-266, Nonr - 580(11), University of Florida, Gainesville.

Simmons, J. P., \& Simonsohn, U. (2017). Power Posing: P-Curving the evidence. Psychological Science, 1-7. https://doi.org/10.1177/0956797616658563

Slepian, M. L., \& Ambady, N. (2012). Fluid movement and creativity. Journal of Experimental Psychology: General, 141(4), 625-629. https://doi.org/10.1037/a0027395

Sligte, D. J., de Dreu, C. K. W., \& Nijstad, B. A. (2011). Power, stability of power, and creativity. Journal of Experimental Social Psychology, 47(5), 891-897. https://doi.org/10.1016/j.jesp.2011.03.009

Smith, K. M., \& Apicella, C. L. (2017). Winners, losers, and posers: The effect of power poses on testosterone and risk-taking following competition. Hormones and Behavior, 92, 172-181. https://doi.org/10.1016/j.yhbeh.2016.11.003 
Smith, P. K., \& Trope, Y. (2006). You focus on the forest when you're in charge of the trees: Power priming and abstract information processing. Journal of Personality and Social Psychology, 90, 578-596. https://doi.org/10.1037/0022-3514.90.4.578

Stanciu, M. M. (2015). Embodied creativity: A critical analysis of an underdeveloped subject. Procedia - Social and Behavioral Sciences, 187, 312-317. https://doi.org/10.1016/j.sbspro.2015.03.058

Taft, R., \& Rossiter, J. R. (1966). The Remote Associates Test: Divergent or Convergent Thinking? Psychological Reports, 19(3), 1313-1314. https://doi.org/10.2466/pr0.1966.19.3f.1313

Torrance, E. P. (1974). The Torrance Tests of Creative Thinking-Norms-Technical Manual Research Edition-Verbal Tests, Forms A and B-Figural Tests, Forms A and B. Princeton, NJ: Personnel Press.

Urban, K. K., \& Jellen, H. G. (1996). Test for creative thinking - Drawing production (TCT$D P)$. Lisse, Netherlands: Swets \& Zeitlinger.

Vohs, K. D., Redden, J. P., \& Rahinel, R. (2013). Physical order produces healthy choices, generosity, and conventionality, whereas disorder produces creativity. Psychological Science, 24, 1860-1867. https://doi.org/10.1177/0956797613480186

Webb, M. E., Little, D. R., \& Cropper, S. J. (2016). Insight is not in the problem: Investigating insight in problem solving across task types. Frontiers in Psychology, 7 (1424). https://doi.org/10.3389/fpsyg.2016.01424 
Weisberg, R. W. (1995). Prolegomena to theories of insight in problem solving: A taxonomy of problems. In R. J. Sternberg \& J. E. Davidson (Eds.), The nature of insight. Cambridge, MA: MIT Press.

Witkin, H., Oltman, P., Raskin, E., \& Karp, S. (1971). Manual: Embedded Figures Test; Children's Embedded Figures Test; and Group Embedded Figures Test. Palo Alto, CA: Consulting Psychologists Press, Inc.

Yap, A. J., Wazlawek, A. S., Lucas, B. J., Cuddy, A. J. C., \& Carney, D. R. (2013). The ergonomics of dishonesty: the effect of incidental posture on stealing, cheating, and traffic violations. Psychological Science, 24, 2281-2289. https://doi.org/10.1177/0956797613492425

Yin, Y., \& Smith, P. (2020). Power and cognitive functioning. Current Opinion in Psychology, 33, 95-99. https://doi.org/10.1016/j.copsyc.2019.07.020

Zhou, Y., Zhang, Y., Hommel, B., \& Zhang, H. (2017). The impact of bodily states on divergent thinking: Evidence for a control-depletion account. Frontiers in Psychology, 8, 1546. https://doi.org/10.3389/fpsyg.2017.01546

Appendix. Expansive and contractive postures used in the leaflets in all studies. 


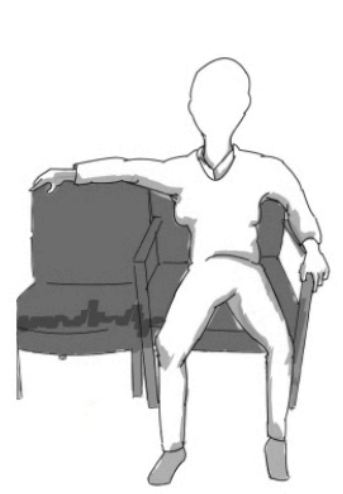

A

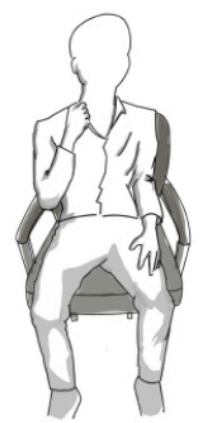

A

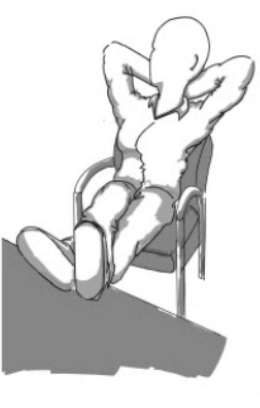

B

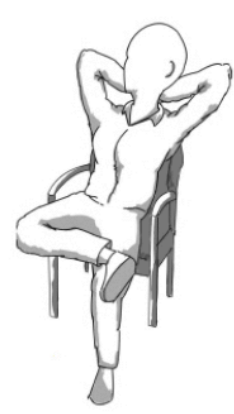

C

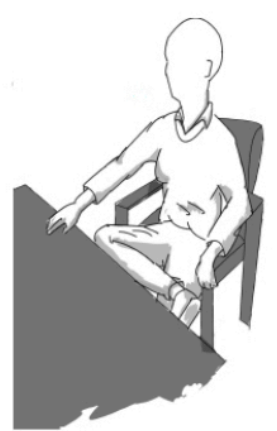

D

Contractive postures

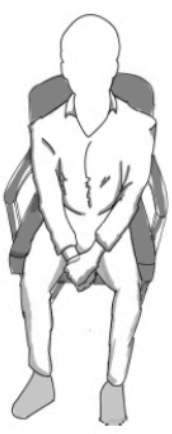

B

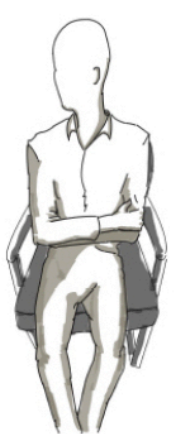

C

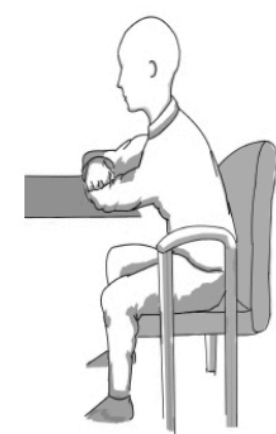

D

\section{Footnotes}

\footnotetext{
${ }^{1}$ A pretest was carried out in which 63 participants (56 females and 7 males) were presented with illustrations of either four expansive or four contractive postures and rated the perceived comfort, difficulty, pain, and power of each on a 7-point Likert scale from 1 (very low) to 7 (very high). Using a two-tailed test, results revealed no differences between expansive and contractive postures on comfort (mean difference $=-0.06,95 \%$ confidence interval, or $\mathrm{CI}=[-$ $0.528,0.409]), t(61)=-0.255, p=.80$, Cohen's $d=0.064$, difficulty (mean difference $=$ -
} 
$0.317,95 \%$ confidence interval, or $\mathrm{CI}=[-0.821,0.188]), t(61)=-1.254, p=.21$, Cohen's $d$ $=0.32$, and pain (mean difference $=-0.449,95 \%$ confidence interval, or $\mathrm{CI}=[-0.961,0.063]$ ), $t(61)=-1.755, p=.084$, Cohen's $d=0.442$. Interestingly, a greater feeling of power was found after presentation of models in expansive postures $(M=3.984$ and $S D=1.143)$ than contractive postures $(M=3.124$ and $S D=0.968), t(61)=-3.221, p=.002$, Cohen's $d=0.812$ (mean difference $=-0.861,95 \%$ confidence interval, or $\mathrm{CI}=[-1.395,0.326]$ ). Paired-samples t-tests did not yield significant differences in the feeling of power between the postures in each condition.

${ }^{2}$ The same short questionnaire was administered at the end of the creativity task in all studies. Three items were used to evaluate the participant's confidence about the task on a 7-point Likert scale ranging from 1 (totally disagree) to 7 (totally agree): I felt confident when carrying out the task; I was sure of myself while carrying out the task; I did not feel up to this task (reverse scored) (Cronbach's alpha $=.63$ ). Three other items were used to evaluate the participant's approach motivation on a 7-point Likert scale ranging from 1 (totally disagree) to 7 (totally agree): I focused on the task; I'm afraid I did not have enough ideas during the task; I don't think I was sufficiently attracted by the task (the last two items were reverse scored) (Cronbach's alpha $=.30)$. As the reliability was low, we have not included details of the results in our studies.

${ }^{3}$ As the original version of the RAT contains 30 items, we also performed statistical analyses on 30 items instead of 38, removing the last eight difficult items where the solution rate ranged from 1.22 to $8.54 \%$. Results revealed that the number of correct items was higher in the contractive posture condition $(M=18.07$ and $S D=4.916)$ than in the expansive posture condition $(M=14.80$ and $S D=4.696$; mean difference $=3.268,95 \%$ confidence interval, or $\mathrm{CI}$ $=[1.157,5.379]), t(80)=3.081, p=.003$, Cohen's $d=0.681$.

${ }^{4}$ Measures of feeling of power and recognition from Amy Cuddy's (2012) TED talk on "power posing" were added in the fifth study. As the TED talk has received more than 58 million views to date, participants were shown a photo of Amy Cuddy giving her TED talk and asked if they recognized her (see Ronay et al., 2017, for details). The feeling of power was measured with three items (I felt a feeling of power, I thought I was controlling the situation, I felt powerful, Cronbach's alpha $=.83$ ), and no difference was observed between the contractive $(M=3.167)$ and the expansive posture conditions $(M=2.905), t(82)=0.827$, $p=.205$. None of the participants said they had seen the TED talk or recognized the photo of Amy Cuddy. 
